\title{
Use of fuzzy cognitive maps for enhanced interaction with multiple mobile devices
}

\author{
Przemysław Kucharski, Dawid Sielski, Tomasz Jaworski, Andrzej Romanowski, Jacek Kucharski \\ Institute of Applied Computer Science \\ Lodz University of Technology \\ ul.Stefanowskiego 18/22, 90-924 Łódź, Poland \\ Email: pkuchars@iis.p.lodz.pl
}

\begin{abstract}
The aim of this work was to design and implement a mechanism supporting collaborative sensemaking in a system of multiple mobile devices implementing spatial awareness. The design is based on an observation how people tend to manage and organize information in physical space. The developed mechanism attempts to determine the relation between atomic elements of information basing on relative position of these elements in time. The proposed solution is based on a simple Fuzzy Inference System and the theory of Fuzzy Cognitive Maps. Physically, the system was implemented for three tablets, for which spatial awareness is simulated with the use of motion tracking system. The system was evaluated in a user study.
\end{abstract}

\section{INTRODUCTION}

$\mathbf{I}$ $\mathrm{N}$ the modern world, human beings and technology have to coexist on daily basis. One of the aspects of this coexistence is how people perceive information using technology. As life is getting faster, the amount of information reaching a person is increasing. Due to that, the mechanisms used to absorb all this information have to become more and more effective. When people are facing information, they have to somehow perceive and understand what this information means - this process is called sensemaking, from "making sense" of information. There exist many techniques to support this process - one of most common examples is underlining the important parts in a text - it simplifies cognition and hierarchizes the information. Another habit many people have, a technique that underlies the following work, is using physical space to support sensemaking - for instance, a student learning for an exam puts the notes in a certain order on the floor, creating a physical space of knowledge - they may guess what each pile of notes contains basing on where it is on the floor. As it was stated, nowadays people use technology, e.g. mobile devices as smartphones and tablets, to explore data and deal with information. There is an emerging need to create technological solutions that will be able to support data exploration processes such as sensemaking. This work is another step forward to obtaining solutions that will help people understand information they see. It was initially inspired by the pace of experiments in this field, and is basing on the earlier step in the work âĂŞ a multi-device, spatially-aware interface that was designed to support sensemaking. The goal of this project is to design and develop mechanism for analysis of spatial data management in multi-device system. The mechanism is based on a complete weighted graph. The design is inspired by the theory of Fuzzy
Cognitive Maps (FCM). The graph reflects mutual relations between atomic elements of information in a set of hints given to solve a problem.

\section{A. Motivation}

The pace of world development poses new challenges in the field of Human-Computer Interaction. People nowadays use mobile devices at all times, and it is not strange to spot a person carrying more than one of them, for example a tablet and a smartphone. It is also more frequent to use mobile devices during meetings, so there is a need for solutions in the field of collaborative sensemaking. The previous work, focusing on the use of spatial awareness in an interface for mobile devices, gave promising results for further development [1]. In the course of work in this area, an idea of implementing the methods of artificial intelligence arose.

\section{RELATED WORK}

The following work covers the areas of data exploration, in particular collaborative sensemaking. It also benefits from the preceding work and insights in the fields of multi-device environments and spatially-aware systems. The original approach, consisting of implementation of AI methods, bases on the theory of Fuzzy Cognitive Maps.

\section{A. Data exploration}

As stated before, technological development requires addressing new challenges in the field of gaining knowledge and understanding the information. One of important issues is how to answer the situation of data exploration in everyday situations. As one of the aspects of ad-hoc data exploration, Fjeld et al. [2] raised the issue of "big data" present in public space - which means that the load of information in this area is rapidly increasing. What lays in connection to that, a considerable part of this information load may be inaccessible to most people. They considered possible designs for ad-hoc data exploration and proposed tangible tabletops as one of possible solutions, with an insight of future technology development for interactive environments. However, this solution assumes introduction of a new equipment to public spaces, where they can serve as data exploration tools. On the other hand, Weise et al. [3] investigated how the issue of data administration, exploration and analysis should be addressed in the age of 
ubiquitous computing. They suggested connecting it to the local infrastructure, both in terms of human awareness and environmental sensing.

\section{B. Multi-device environments}

As it was mentioned, situations when users have several mobile devices to work at the same time can often be encountered. Currently available applications, however, do not provide solutions by which users can benefit from the interactions between the devices they use, i.e. cross-device interactions. The subject of multi-device environments was considered for a long time. Bilezikjian et al. [4] explored how the interactions with handheld devices may look like, although they were not commercially available. Blackwell et al. [5] investigated the issue of tangibility in the context of mobile devices. Cauchard et al. [6] discussed the concerns and opportunities for visual aspect of mobile multi-display environments. In Conductor [7], Hamilton and Wigdor presented a framework for examining the scenarios of crossdevice interactions. They provide functionalities to split the aspects of performed task between several mobile devices. They also elaborated possible usage scenarios for the system, demonstrating their way of understanding cross-device interactions in task-specific domain. The study performed using the developed multi-device system demonstrated that using several connected devices is highly useful to perform certain tasks. Most of the participants made use of multiple devices and of the functionalities enabling them to easily transfer information across devices. The Pass-them-around [8], [9] system developed by Lucero et al. showed that providing people with the functionality of sharing content between their personal devices may lead to enrichment of interactions between users. Cassens et al. [10] proposed a taxonomy for the term cross-device interactions. They introduced the dimensions of ownership, distance and access to classify those interactions. They discussed this classification in the context of the work published in this field. Finally, they proposed a definition: "Cross-device interaction (XDI) is the type of interaction, where human users interact with multiple separate input and output devices, where input devices will be used to manipulate content on output devices within a perceived interaction space with immediate and explicit feedback." In general, the past research in the field of multiple device ecologies demonstrates that once cross-device interactions are implemented and enabled, users adapt to the new environment and benefit from these interactions. This proves, as it was raised in many of the papers, that further exploration of this field is necessary and promises a valuable contribution to our future life.

\section{Spatially-aware systems}

Space and awareness of space is of great importance for human perception of surrounding world. Hall developed a theory describing the relation between spatial arrangement of people and their social behaviours and emotions. Chen and Kotz [11] included spatial awareness as an important aspect of context-aware computing. Some past research show that space may also have a vital impact on understanding and learning processes [12]. There was also some investigation concerning how transferring the perception of space to technology may help users interact with data.

In MochaTop [13], Wo̊̊žniak et al. made a step forwards to understand how spatial combinations of two devices can be used for data exploration. They focused on a single user scenario for ad-hoc interactions, basing on an assumption that a couple of a smartphone and a tablet is more and more often carried by users. They designed and implemented several spatial-based interactions for exploring complex structures of data.

Their study showed that users find it useful to make space one of the input sources in data manipulation. Spindler [14], [15], [16] proposed a way of extending the interaction space into the third dimension. In this work, users are in disposition of several spatially-aware tangible displays to interact with virtual objects present on a central tabletop. This work demonstrated the potential of employing explorable 3D space for visualizations. By direct translation of spatial position to visualization input, he created a tool for intuitive exploration of several types of complex data sets.

The field of extending the interaction space of one mobile device was also investigated. AD-binning [17], which is an abbreviation from Around-Device Binning, is a mobile user interface that allows users organize data spatially outside of the device space. The concept consists of creating virtual zones (bins) around the device. The system tracks relative position of user's finger and the device and thus makes it possible to put pieces of information from the screen into these zones. The motivation of designing such solutions is, as stated by the authors, extending the interaction space due to insufficiency of that space on the screen of a mobile device. The premise that the design of user interfaces for multiple mobile devices should be based on spatial awareness of those devices was discussed by Rädle et al. [18]. They divided cross-device interactions into three groups: (i) spatially agnostic, (ii) based on synchronous gestures and (iii) spatially-aware. Spatially agnostic interactions may be menu- based with some kinds of user-perceivable device identification. Interactions based on synchronous gestures are triggered by simultaneous interaction with two of more devices. Eventually, spatially-aware interactions are based on relative arrangement of devices. The study conducted to compare them demonstrated that in most cases, spatially-aware interactions between devices are expected by the users. However, similarly to the Ballendat's conclusion about proxemics, Rädle et al. clearly emphasise that the way in which spatially-aware interactions should be designed is not yet fully explored and requires further investigation.

1) Hardware for spatial awareness: There are many attempts to find technical solutions for spatial awareness of mobile devices. There are approaches that may benefit both the further examination of spatial interactions, as well as those which may be applied in real world.

HuddleLamp [19] provides a solution for creating ad-hoc 
multi-device ecologies on a tabletop based on video processing. Rädle et al. propose spatially-aware multiple device systems as an alternative for interactive tabletops. An interesting approach in this work is tracking not only the devices, but also the motion of users' hands to enrich interactions. One of technical possibilities that may be implemented in commercially available devices was presented by Ellyptic Labs, with a ultrasound Doppler-based system for spatial awareness of mobile devices. ${ }^{1}$ Another emerging direction is embedding the sensing in the environment. An effort was made recently to develop tomography-based table for spatial positioning of devices [20]. This solution is based on the change in electrical capacitance triggered by mobile devices, which usually consist of magnetic elements. This approach gives promising results from the very beginning, yet it is in early stage of development.

\section{Fuzzy logic}

Fuzzy logic is a form of mathematical logic where the variables may not only be 0 or 1 , which is true or false, but may be any real number from this interval[21]. This makes it possible to mathematically introduce the concept of something being partially true. For linguistic variables, a membership function can be introduced to manage the degree of truth[22]. This membership functions characterize various sub-ranges of a continuous variable. By the process of fuzzification, it is possible to map the input value of a variable to its membership function, and therefore obtain a more accurate result than with the use of crisp logic. The concept of fuzzy logic is used in many applications. For instance, Zadeh [23] proposed a methodology for computations using very imprecise information, where words are used instead of numbers. This approach requires the use of fuzzy logic and fuzzy set and promises very effective solutions for many problems. Another very interesting work in the field of fuzzy logic and sets, fitting closely to the scope of this project, is the theory of fuzzy information granulation proposed by Zadeh [24]. This work elaborates the human reasoning system in the point of view of automatisation using fuzzification and granulation.

\section{E. Fuzzy cognitive maps}

The concept of Fuzzy Cognitive Maps was proposed by Kosko [25] as a structure to represent causal reasoning. The theory is based on an observation that in knowledge processing, most of the relations, including classification and causality, are uncertain and imprecise. Fuzzy Cognitive Maps are graph structures, where the nodes of the graph represent causal objects or concepts, and the edges represent mutual relation between these concepts. The concept of FCM is based on the theory of cognitive maps introduced by Polish scientist Axelrod [26] for representing social scientific knowledge. Papageorgiou [27] stated that "Fuzzy cognitive maps (FCMs) are a modeling methodology based on exploiting knowledge and experience." This concept has been successfully applied in many fields, including political decisions [28], modeling

${ }^{1}$ www.ellypticlabs.com electrical circuits [29] or organisational behaviour [30]. Most of the implementations of FCMs require expert in the process of map design, but Aguilar reveals in his survey [31] that there are attempts to develop FCMs automatically from raw data. This survey also shows a wide range of possible applications, covering both causal and non-causal relationships with imprecise information.

\section{THEORETICAL BACKGROUND}

The design and implementation of the proposed mechanism poses several challenges. The first is the determination of mutual position of two atomic elements of information in the space. This is possible thanks to the motion tracking system, which sends the position of each device configured as a rigid prop. The information given by the system is translation versus the origin of global coordinate system and the rotation in Euler angles. On the other side, the device sends the position of a post-it on its screen. Basing on this, a matrix calculus enables determining the position of each post-it in the global coordinate system.

The most important part is the model of the data set, containing information about the mutual relation between set elements. A concept responding well to the needs of this project is Fuzzy Cognitive Map. Therefore, the entire data set was modeled in the system in a form of complete weighted graph. Some implementations of FCM are used to describe causal relations, and for this purpose directed graphs are used. In the case of this design, the aim was simply to reflect the strength of mutual relation between each two elements âĂŞ the atomic elements of information are represented as graph nodes, and the weight of the edge connecting two nodes represents the aforementioned strength of relation.

In this model, it was also extremely important to reflect the imprecise nature of the relations. This was obtained by using FCM, not the original concept of Cognitive Maps. In the model, the weight of the node is any number from 0 to 1 , where 0 âĂŞ no relation, 1 âĂŞ strong relation. This makes it possible to show the user not only which elements are related, but also give them hints which of them are related stronger than others.

\section{MOdELling THE RELATIONS}

The class InteractionModel manages all the position data concerning both the devices and the resources in form of textual hints. The original approach in using this positional information is used to determine the numerical values in the weighted graph. A complete weighted graph containing 31 nodes is implemented in form of an array. The positions of each resource in global coordinate system are updated basing on data from tablets and motion tracking. In order to do that, Java library used for matrix calculus is used. The server calculates the distance between the resources. The distance is normalized to scale $0-10$ basing on maximum distance in the current step. Then, the normalized distance is passed to mechanism that uses fuzzy logic. To implement fuzzy logic rules, a Java library jFuzzyLogic [32], [33] was implemented 
in the project. It uses Fuzzy Control Language to implement the fuzzy system and a interface to use the system in Java. The output variable, which is the change in relation, is returned after evaluation of the system. The weights in the graph are changed according to the response of fuzzy system, and then the weights in the entire graph are normalized to interval 01 , basing on the maximum and minimum value of relation found in the graph. If the user chooses to display relations of one of the post-its by clicking it, the InteractionModel returns the related post-its (i.e. which have relation strength with the chosen one different from 0) along with the weight of the graph edge.

\section{STUDY AND RESUlTS}

The method of evaluation of the developed solution is a user study. User study is a well known method in HumanComputer Interaction. It is a way to experimentally test a hypothesis. It can test a variety of measures, for example user experience, efficiency, accuracy, in general both qualitative and quantitative performance of a system. There are several procedures of planning and running user studies [34]. In this project, two systems are tested to determine the potential difference in their performance from the point of view of a user:

- A system supporting static relations between elements

- A system supporting adaptive relations between elements based on interaction of the user

\section{A. Experimental procedure}

The hypothesis of the study is that the system offering adaptive relation feedback may have a positive impact on the effectiveness of the solution. The study is divided into two parts: in one, the study apparatus is the simplified version of the system with static relations to have baseline results, in the other one, current implementation is used in order to measure the differences. The study in each case consist of several parts. In both stages, the system was set up in an isolated environment. Participants were informed on the purpose of the study and instructed on the task and how to use the system. Then they were asked to solve the mystery. The time for solving the mystery was approximately $30 \mathrm{~min}$. After that, they were shortly debriefed on the functionalities of the system and overall opinion about the proposed solution.

\section{B. Task description}

The task in the study was to solve a crime mystery. In the data set, there were 31 hints containing information about places, events and people. The questions that were to be answered included: the person of murderer, the time of murder, the place and the motive. The preliminary fact is that $\mathrm{Mr}$ Kelley was murdered. The questions to be answered involve the person who killed, the place, time and the motive of murder. The structure of the task is complicated: there are several people, some of which have nothing to do with the crime under investigation. The evidence is uncertain and the information about the place inconsistent. To show the difficulty of the task, it is enough to quote the prepared answer: "After receiving a superficial gunshot wound from $\mathrm{Mr}$. Jones, $\mathrm{Mr}$. Kelley went to Mr. Scott's apartment where Mr. Scott killed him with a knife at 12:30 AM because Mr. Scott was in love with Mr. Kelley's wife". The study task was based on a criminal mystery from a book prepared for teaching collaboration skills [35].

\section{Study results: static relations system}

In the first stage of study, with the version of the system supporting static relations established among atomic information elements, $n=16$ participants in 8 pairs (aged 24-61, $\mu=37$, 9 males, 7 females) were asked to complete the task (Figure 1). They completed the task in mean Task Completion Time (TCT) of 21 minutes and 18 seconds, with standard deviation of $3 \mathrm{~min} 55 \mathrm{sec}$. At this stage, the relations in the system were predetermined, basing only on text analysis of the hints. The system supported highlighting related hints and displaying a time line - an ordered connection between hints containing temporal information.

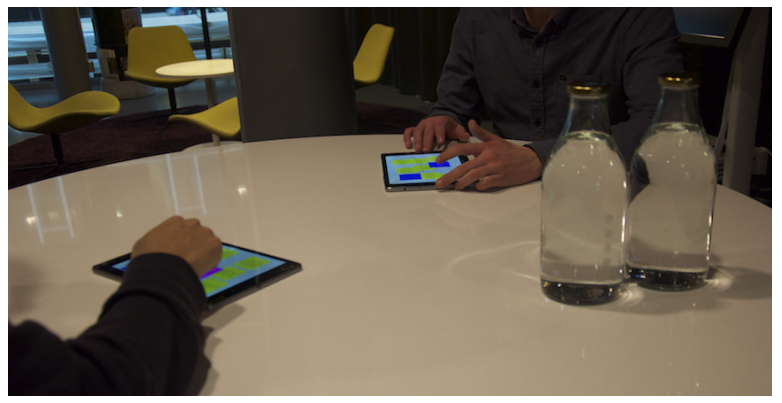

Fig. 1. Static relations version of the system

\section{Study results: adaptive relations system}

The second stage of the study was performed with the use of current implementation of the system. In this study, $n=8$ participants in 4 pairs (aged $15-23, \mu=21,5$ males, 3 females) were asked to solve the crime mystery (Figure 2). For the purpose of this stage, the same crime mystery with exactly the same hints was used. Participants came with the proper response in mean time of 19 minutes 41 seconds, with standard deviation of $4 \mathrm{~min} 49 \mathrm{sec}$.

\section{E. Discussion of the results}

As the main quantitative measure of the results the Mean Task Completion Time (MTCT) was chosen, which expresses the average TCT achieved by pairs in each study stage. The results of the performed study reveal a slight difference in MTCT in favor of the new system (Table I). It is difficult to say basing on this results whether or not this difference is statistically significant. However, basing on simple analysis of the obtained times, it can be stated that the direction chosen in the development of the system, consisting of applying AI methods such as fuzzy logic and FCM is proper and should 


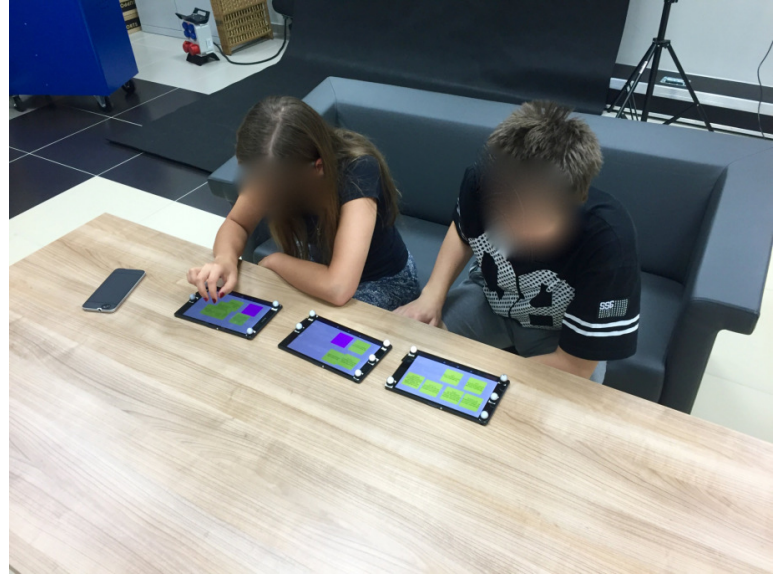

Fig. 2. Adaptive relations version of the system

TABLE I

RESULTS OF BOTH STUDIES

\begin{tabular}{|l|l|l|l|l|}
\hline \multirow{2}{*}{ System } & \multicolumn{2}{|c|}{ MTCT } & \multicolumn{2}{c|}{$\sigma_{T C T}$} \\
\cline { 2 - 5 } & min & sec & min & sec \\
\hline 1 & 21 & 18 & 3 & 55 \\
\hline 2 & 19 & 41 & 4 & 49 \\
\hline
\end{tabular}

be continued. Apart from the quantitative results, qualitative results obtained during interviews with participant is also very important at this stage of development. This qualitative results were received from the analysis of recordings, user interviews and questionnaires. At both stages, there were participants that were distanced from such new technological solution, however they recognized the potential advantages of such systems. There are also many voices concerning the decision on implementing such solution on tablets instead of, for instance, one big tactile table. This is actually a discussion beyond this project, because people working on several tablets are more likely to meet in ad-hoc real-life situations than around a huge interactive tabletop. In the current implementation of the system, users generally agreed with the idea of determining relations, and they noticed the intuitive way of displaying it to the user in form of different, more light or dark, shades of one color. The idea of such a solution arose on the basis of preliminary study, where participants were asked to complete the task using pieces of paper. An observation was made then on how people use physical space to organize information. This subject was further investigated and the preliminary ideas for design were published in a workshop paper. This triggered the development of a system based on Fuzzy Inference System and Fuzzy Cognitive Maps. The solution presented in this work is another step towards supporting human cognition with the use of modern technology. The system translates the mutual spatial position of atomic elements of information, which are hints leading to solution in a crime mystery, into their mutual relation, and afterwards allows the user to display these relations, which should enhance the process of making sense of information.

\section{F. Directions for future work}

The main direction for future work on this project is an attempt to determine the parameters of the system - both in terms of FIS and FCM. This means further study how exactly should the relation change basing on the input data to give best results. Another area of further development is to find which other information that can be obtained from the analysis of spatiotemporal data in the system should have an impact on the strength of relation. The latter thing mentioned is very intriguing both from theoretical and experimental point of view. It also leads to the last, but not least planned future stage of development of the system. This should be an attempt to generalize the solution to a wide range of problems - so that it can support the sensemaking process with minimal expert knowledge in terms of initial state and parameters of the system.

\section{CONCLUSIONS}

In the course of this work, a mechanism supporting collaborative sensemaking with multi-device spatially-aware system was elaborated. This work was preceded by profound research in the field of user interfaces in multi-device environments and was mainly motivated by an emerging need of implementing advanced processing methods in HCI. The results of the user study, compared with the results obtained with the system which did not support adapting the mutual relations basing on user interaction, give a promising insight into the future of such solutions and prove that this step already taken is a step in the right direction. This work needs to and will be further developed.

\section{REFERENCES}

[1] P. Wozniak, N. Goyal, P. Kucharski, L. Lischke, S. Mayer, and M. Fjeld, "RAMPARTS," in Proceedings of the 2016 CHI Conference on Human Factors in Computing Systems - CHI '16, (New York, New York, USA), pp. 2447-2460, ACM Press, 2016.

[2] M. Fjeld, P. Woźniak, J. Cowls, and B. Nardi, "Ad hoc encounters with big data: Engaging citizens in conversations around tabletops," First Monday, vol. 20, 22015.

[3] S. Weise, J. Hardy, P. Agarwal, P. Coulton, A. Friday, and M. Chiasson, "Democratizing ubiquitous computing," in Proceedings of the 2012 ACM Conference on Ubiquitous Computing - UbiComp '12, (New York, New York, USA), p. 521, ACM Press, 92012.

[4] M. Bilezikjian, S. R. Klemmer, R. L. Mandryk, J. A. Landay, and L. M. Inkpen, "Exploring a New Interaction Paradigm for Collaborating on Handheld," 82002.

[5] A. F. Blackwell, G. Bailey, I. Budvytis, V. Chen, L. Church, L. Dubuc, D. Edge, M. Linnap, V. Naudziunas, and H. Warrington, "Tangible Interaction in a Mobile Context," CHIâAZŹ07 Workshop on Tangible User Int. in Context and Theory, 2007.

[6] J. R. Cauchard, M. Löchtefeld, P. Irani, J. Schoening, A. Krüger, M. Fraser, and S. Subramanian, "Visual separation in mobile multidisplay environments," in Proceedings of the 24th annual ACM symposium on User interface software and technology - UIST'11, (New York, New York, USA), p. 451, ACM Press, 102011.

[7] P. Hamilton and D. J. Wigdor, "Conductor: enabling and understanding cross-device interaction," in Proceedings of the 32nd annual ACM conference on Human factors in computing systems - CHI '14, (New York), pp. 2773-2782, ACM Press, 42014.

[8] A. Lucero, J. Holopainen, and T. Jokela, "Pass-them-around: collaborative use of mobile phones for photo sharing," in Proceedings of the 2011 annual conference on Human factors in computing systems - CHI '11, (New York), p. 1787, ACM Press, 52011. 
[9] A. Lucero, T. Jokela, A. Palin, V. Aaltonen, and J. Nikara, "EasyGroups: binding mobile devices for collaborative interactions," in Proceedings of the 2012 ACM annual conference extended abstracts on Human Factors in Computing Systems Extended Abstracts - CHI EA '12, (New York), p. 2189, ACM Press, 52012.

[10] J. Cassens, "Cross-Device Interaction," in AMBIENT 2013 : The Third International Conference on Ambient Computing, Applications, Services and Technologies, 2013.

[11] G. Chen and D. Kotz, "A survey of context-aware mobile computing research," 2000.

[12] D. Reilly, A. Echenique, A. Wu, A. Tang, and W. K. Edwards, "Mapping out Work in a Mixed Reality Project Room," in Proceedings of the 33rd Annual ACM Conference on Human Factors in Computing Systems CHI '15, (New York, New York, USA), pp. 887-896, ACM Press, 4 2015.

[13] P. W. Wozniak, B. Schmidt, L. Lischke, Z. Franjcic, A. E. Yantaç, and M. Fjeld, "MochaTop," in Proceedings of the extended abstracts of the 32nd annual ACM conference on Human factors in computing systems CHI EA '14, (New York, New York, USA), pp. 2329-2334, ACM Press, 42014.

[14] M. Spindler, W. Büschel, C. Winkler, and R. Dachselt, "Tangible displays for the masses: spatial interaction with handheld displays by using consumer depth cameras," Personal and Ubiquitous Computing, vol. 18, pp. 1213-1225, 112013.

[15] M. Spindler and R. Dachselt, "Exploring information spaces by using tangible magic lenses in a tabletop environment," Proceedings of the 28 th of the international conference extended abstracts on Human factors in computing systems - CHI EA '10, p. 4771, 2010.

[16] M. Spindler, "Spatially aware tangible display interaction in a tabletop environment," Proceedings of the 2012 ACM international conference on Interactive tabletops and surfaces - ITS '12, p. 277, 2012.

[17] K. Hasan, D. Ahlström, and P. Irani, "Ad-binning: leveraging around device space for storing, browsing and retrieving mobile device content," Proceedings of CHI 2013, pp. 899-908, 2013.

[18] R. Rädle, H.-C. Jetter, M. Schreiner, Z. Lu, H. Reiterer, and Y. Rogers, "Spatially-aware or Spatially-agnostic?," in Proceedings of the 33rd Annual ACM Conference on Human Factors in Computing Systems CHI '15, (New York, New York, USA), pp. 3913-3922, ACM Press, 4 2015.

[19] R. Rädle, H.-C. Jetter, N. Marquardt, H. Reiterer, and Y. Rogers, "HuddleLamp: Spatially-Aware Mobile Displays for Ad-hoc Around-
the-Table Collaboration," in Proceedings of the Ninth ACM International Conference on Interactive Tabletops and Surfaces - ITS '14, (New York), pp. 45-54, ACM Press, 112014.

[20] P. Kucharski, A. Romanowski, K. Grudzień, and P. Woźniak, "TomoSense: Towards Multi-Device Spatial Awareness Based on Independent Plane Sensing." 2016.

[21] P. Hájek, Metamathematics of fuzzy logic. Kluwer, 1998.

[22] E. Cox, The fuzzy systems handbook : a practitioner's guide to building and maintaining fuzzy systems. AP Professional, 1994.

[23] L. Zadeh, "Fuzzy logic = computing with words," IEEE Transactions on Fuzzy Systems, vol. 4, pp. 103-111, 51996.

[24] L. A. Zadeh, "Toward a theory of fuzzy information granulation and its centrality in human reasoning and fuzzy logic," Fuzzy Sets and Systems, vol. 90, pp. 111-127, 91997.

[25] B. Kosko, "Fuzzy cognitive maps," International Journal of ManMachine Studies, vol. 24, pp. 65-75, 11986.

[26] R. Axelrod, "The cognitive mapping approach to decision making," Structure of decision, pp. 221-250, 1976.

[27] E. I. Papageorgiou and C. D. Stylios, "Fuzzy cognitive maps," Handbook of Granular Computing, pp. 755-774, 2008

[28] R. Taber, "Knowledge processing with Fuzzy Cognitive Maps," Expert Systems with Applications, vol. 2, no. 1, pp. 83-87, 1991.

[29] M. Styblinski and B. Meyer, "Fuzzy cognitive maps, signal flow graphs, and qualitative circuit analysis," in IEEE International Conference on Neural Networks, pp. 549-556, IEEE, 1988.

[30] J. Craiger, D. Goodman, R. Weiss, and A. Butler, "Modeling organizational behavior with fuzzy cognitive maps," vol. 1, pp. 120-123, 1996

[31] J. Aguilar, "A survey about fuzzy cognitive maps papers," International journal of computational cognition, vol. 3, no. 2, pp. 27-33, 2005.

[32] P. Cingolani and J. Alcala-Fdez, "jFuzzyLogic: a robust and flexible Fuzzy-Logic inference system language implementation," in Fuzzy Systems (FUZZ-IEEE), 2012 IEEE International Conference on, pp. 1-8, IEEE, 2012.

[33] P. Cingolani and J. Alcalá-Fdez, "jFuzzyLogic: a Java Library to Design Fuzzy Logic Controllers According to the Standard for Fuzzy Control Programming,"

[34] K. Hornbæk, "Some whys and hows of experiments in human-computer interaction," Human-Computer Interaction, vol. 5, no. 4, pp. 299-373, 2011.

[35] G. Stanford and B. D. Stanford, Learning Discussion Skills Through Games. Citation Press, 1971. 